\title{
A RESTRICTION THEOREM FOR SEMISIMPLE LIE GROUPS OF RANK ONE
}

BY

JUAN A. TIRAO

\begin{abstract}
Let $\mathfrak{g}_{\mathbf{R}}=\mathfrak{f}_{\mathbf{R}}+\mathfrak{p}_{\mathbf{R}}$ be a Cartan decomposition of a real semisimple Lie algebra $\mathfrak{g}_{\mathbf{R}}$ and let $\mathfrak{g}=\mathfrak{f}+\mathfrak{p}$ be the corresponding complexification. Also let $\mathfrak{a}_{\mathbf{R}}$ be a maximal abelian subspace of $\mathfrak{p}_{\mathbf{R}}$ and let $a$ be the complex subspace of $\mathfrak{p}$ generated by $\mathfrak{a}_{\mathbf{R}}$. We assume $\operatorname{dim} \mathfrak{a}_{\mathbf{R}}=1$. Now let $G$ be the adjoint group of $g$ and let $K$ be the analytic subgroup of $G$ with Lie algebra $\operatorname{ad}_{g}\left({ }^{*}\right)$. If $S^{\prime}(g)$ denotes the ring of all polynomial functions on a then clearly $S^{\prime}(g)$ is a $G$-module and a fortiori a $K$-module. In this paper, we determine the image of the subring $S^{\prime}(g)^{K}$ of $K$ invariants in $S^{\prime}(g)$ under the restriction map $\left.f \mapsto f\right|_{\mathfrak{t}+\mathfrak{a}}\left(f \in S^{\prime}(\mathfrak{g})^{K}\right)$.
\end{abstract}

1. Introduction. Consider a reductive Lie algebra $\mathfrak{g}_{\mathbf{R}}$ over $\mathbf{R}$, a fixed Cartan decomposition $\mathfrak{g}_{\mathbf{R}}=\mathfrak{f}_{\mathbf{R}}+\mathfrak{p}_{\mathbf{R}}$ and a maximal abelian subspace $\mathfrak{a}_{\mathbf{R}}$ of $\mathfrak{p}_{\mathbf{R}}$. Extend $\mathfrak{g}_{\mathbf{R}}$ to a Cartan subalgebra $\mathfrak{h}$ of the complexification $\mathfrak{g}=\mathfrak{f}+\mathfrak{p}$ of $\mathfrak{g}_{\mathbf{R}}$ in the usual way. By $\phi$ we shall denote the set of roots of the pair $(\mathfrak{g}, \mathfrak{h})$ and by $W_{\phi}$ the corresponding Weyl group, whereas $\Delta$ will denote the set of roots of the pair $\left(\mathfrak{g}_{\mathbf{R}}, \mathfrak{a}_{\mathbf{R}}\right)$ and $W_{\Delta}$ the corresponding Weyl group. Let $G$ be the adjoint group of $\mathfrak{g}$ and let $K$ be the analytic subgroup of $G$ with Lie algebra $\operatorname{ad}_{g}(\mathfrak{F})$. If $H$ is a group and $V$ a finite dimensional $H$-module over $\mathrm{C}$, let $S^{\prime}(V)$ denote the ring of all polynomial functions on $V$ and let $S^{\prime}(V)^{H}$ denote the subring of $H$ invariants.

Fundamental for many questions in representation theory is the following Chevalley's Restriction Theorem:

(i) The operation of restriction from $\mathfrak{g}$ to $\mathfrak{h}$ induces an isomorphism of $S^{\prime}(\mathfrak{g})^{G}$ onto $S^{\prime}(\mathfrak{h})^{W_{\phi}}$;

(ii) The operation of restriction from $\mathfrak{p}$ to a induces an isomorphism of $S^{\prime}(\mathfrak{p})^{K}$ onto $S^{\prime}(a)^{W_{\Delta}}$.

Also we have a theorem of the same nature due to Helgason: If $\mathfrak{g}_{\mathbf{R}}$ is a classical semisimple Lie algebra (with real or complex structure) then the restriction from $\mathfrak{h}$ to a maps $S^{\prime}(\mathfrak{h})^{\mathrm{w}_{\phi}}$ onto $S^{\prime}(\mathfrak{a})^{\mathrm{w}_{\Delta}}$. This does not hold in general for the real forms of the exceptional Lie algebras $E_{6}, E_{7}, E_{8}$, but in any event, the elements in $\left.S^{\prime}(a)\right)^{W_{\Delta}}$ are all obtained from rational invariants on $\mathfrak{h}$ by restriction. In fact we have the following result of Harish-Chandra and Helgason: Let $Q\left(S^{\prime}(\mathfrak{h})^{W_{\phi}}\right)$ and $Q\left(S^{\prime}(\mathfrak{a})^{W_{\Delta}}\right)$ denote the quotient fields of $S^{\prime}(\mathfrak{h})^{W_{\phi}}$ and $S^{\prime}(\mathfrak{a})^{W_{\Delta}}$ respectively; then the restriction from $\mathfrak{h}$ to a induces a mapping of $Q\left(S^{\prime}(\mathfrak{h})^{W_{\phi}}\right)$ onto $Q\left(S^{\prime}(\mathfrak{a})^{W_{\Delta}}\right)$ (for all this see $\$ 2.1 .5$ in [4]).

Received by the editors October 24, 1982.

1980 Mathematics Subject Classification. Primary $22 \mathrm{E} 46$.

${ }^{1}$ Partially supported by SUBCYT and CONICET (Argentina) grants.

(C)1983 American Mathematical Society $0002-9947 / 83 \$ 1.00+\$ .25$ per page 
This paper is concerned with the determination of the image of the homomorphism of $S^{\prime}(\mathfrak{g})^{K}$ into $S^{\prime}(\mathfrak{f}+\mathfrak{a})$ induced by restriction from $g$ to $\mathfrak{f}+\mathfrak{a}$. In [3] a suitable element $b \in S^{\prime}(g)^{K}$ is defined and the following theorem of Kostant is proved: Let $S^{\prime}(\mathfrak{g})_{b}^{K}$ be the localization of $S^{\prime}(g)^{K}$ by $b$ (i.e. the ring of all rational functions on $g$ of the form $f / b^{k}$ where $f \in S^{\prime}(\mathfrak{g})^{K}$ and $\left.k \in Z\right)$ and let $S^{\prime}(\mathfrak{f}+a)_{b_{0}}^{M^{\prime}}$ be the localization of $S^{\prime}(\mathfrak{f}+\mathfrak{a})^{M^{\prime}}$ by $b_{0}=b_{\mid \mathfrak{q}+a}, M^{\prime}$ being the normalizer of a in $K$; then the restriction from $g$ to $+a$ induces an isomorphism of $S^{\prime}(g)_{b}^{K}$ onto $S^{\prime}(\mathfrak{f}+a)_{b_{0}}^{M^{\prime}}$.

Starting from this result we are able to characterize the image of $S^{\prime}(g)^{K}$ in $S^{\prime}(\mathfrak{f}+\mathfrak{a})^{M^{\prime}}$ in the split rank one case. Thus, from now on we assume that $\mathfrak{a}_{\mathbf{R}} \cap\left[\mathfrak{g}_{\mathbf{R}}, \mathfrak{g}_{\mathbf{R}}\right]$ is one dimensional. If $M$ is the centralizer of $a$ in $K$ then $M^{\prime} / M \simeq W_{\Delta}$. Let $\mathrm{T}$ denote the set of equivalence classes of irreducible, finite dimensional holomorphic representations of $K$ admitting an $M$-fixed vector. Using results in [2] we prove that any $\tau \in \mathrm{T}$ can be realized as a $K$-submodule of homogeneous harmonic elements in $S(\mathfrak{p})$. The degree of these elements, $d(\tau)$, is uniquely determined by $\tau$. Let $S_{n}^{\prime}(a)$ denote the homogeneous subspace of $S^{\prime}(a)$ of degree $n$, and let $S^{\prime}(\mathfrak{f})_{\tau}$ denote the primary component of $S^{\prime}(\mathfrak{f})$ of type $\tau$. Our main result is the following.

THEOREM. The operation of restriction from $\mathrm{g}$ to + a induces an isomorphism of $S^{\prime}(\mathrm{g})^{K}$ onto

$$
\bigoplus \bigoplus_{n \geqslant 0} \bigoplus_{\substack{\tau \in \mathrm{T} \\ d(\tau) \leqslant n}}\left(S^{\prime}(\mathfrak{f})_{\tau}^{M} \otimes S_{n}^{\prime}(\mathfrak{a})\right)^{W_{\Delta}}
$$

Let $\mathcal{G}, \mathcal{K}$ and $\mathcal{Q}$ denote the universal enveloping algebras over $\mathbf{C}$, of $\mathfrak{g}, \mathfrak{f}$ and $\mathfrak{a}$, respectively. Also let $\mathcal{G}^{K}$ and $\mathcal{K}^{M}$ be the centralizers of $K$ in $\mathcal{G}$ and of $M$ in $\mathscr{K}$, respectively. In many fundamental questions concerning the infinite dimensional representation theory of a Lie group with Lie algebra $g_{R}$ it would be very important to know the image of an injective antihomomorphism $P: \mathcal{G}^{K} \rightarrow \mathcal{K}^{M} \otimes Q$, due to Lepowsky and Rader (see [5 or 6]), which replaces the famous Harish-Chandra homomorphism $\gamma: \mathcal{G}^{K} \rightarrow \mathbb{Q}$ (see [3]). Our main theorem should prove useful in this respect.

2. We use much of the notation in [2]. Thus $\mathfrak{g}_{\mathbf{R}}=\mathfrak{f}_{\mathbf{R}}+\mathfrak{p}_{\mathbf{R}}$ is a Cartan decomposition of a real reductive Lie algebra $g_{\mathbf{R}}$ and $g=\mathfrak{f}+\mathfrak{p}$ is the corresponding complexification. The associated Cartan involution $\theta$ is 1 on $\mathfrak{f}$ and -1 on $\mathfrak{p}$. Also, $\mathfrak{a}_{\mathbf{R}}$ is a maximal abelian subspace of $\mathfrak{p}_{\mathbf{R}}$, so that its complexification $a$ is a Cartan subspace of $\mathfrak{p}$. Let $G$ be the adjoint group of $\mathfrak{g}$ and let $K_{\theta}$ be the subgroup of all elements in $G$ which commute with $\theta$. Clearly and $p$ are stable under the action of $K_{\theta}$. Now if $K$ denotes the analytic subgroup of $G$ with Lie algebra $\operatorname{ad}_{g}(\mathfrak{f})$, then $K$ is the identity component of $K_{\theta}$. Moreover, if $F$ is the set of all elements of order 2 in the connected Lie subgroup $A$ of $G$ corresponding to $\operatorname{ad}_{\mathrm{g}}(a)$, then $F$ is a finite abelian group of order $2^{\operatorname{dim}_{c}(a)}$ which normalizes $K$ and such that $K_{\theta}=K F$ (see Proposition 1, p. 761 in [2]).

For any vector space $V$ let $S^{\prime}(V)$ denote the ring of all polynomial functions on $V$, and for every nonnegative integer $i$ let $S_{i}^{\prime}(V)$ denote the homogeneous subspace of 
$S^{\prime}(V)$ of degree $i$. Then $S^{\prime}(g)$ is a $K_{\theta}$-module: if $f \in S^{\prime}(\mathfrak{g})$ and $a \in K$ then af $\in S^{\prime}(g)$ is given by $(a f)(x)=f\left(a^{-1} \cdot x\right), x \in g$. Let $S^{\prime}(g)^{K}$ be the ring of $K$-invariant polynomials.

The injection map $\mathfrak{t}+\mathfrak{a} \rightarrow \mathfrak{f}+\mathfrak{p}=\mathfrak{g}$ induces contravariantly the restriction homomorphism $S^{\prime}(\mathfrak{g}) \rightarrow S^{\prime}(\mathfrak{f}+\mathfrak{a})$. This homomorphism restricted to $S^{\prime}(\mathfrak{g})^{K}$ induces a homomorphism

$$
\pi: S^{\prime}(\mathfrak{g})^{K} \rightarrow S^{\prime}(\mathfrak{f}+\mathfrak{a}) .
$$

In [3] a homogeneous polynomial $b \in S^{\prime}(g)^{K}$ is defined such that $b(x+y)=b(y)$ for all $x \in \mathfrak{f}$ and $y \in \mathfrak{p}$. If $M^{\prime}$ is the normalizer of $\mathfrak{a}$ in $K$ then $M^{\prime}$ operates on $\mathfrak{f}^{\prime}+\mathfrak{a}$ and we may consider $S^{\prime}(\mathfrak{f}+\mathfrak{a})^{M^{\prime}}$, the ring of $M^{\prime}$-invariant polynomials. Let $S^{\prime}(g)_{b}^{K}$ be the localization of $S^{\prime}(\mathfrak{g})^{K}$ by $b$, so that $S^{\prime}(\mathfrak{g})_{b}^{K}$ is the ring of all rational functions on $g$ of the form $f / b^{q}$ where $f \in S^{\prime}(g)^{K}$ and $q \in \mathbf{Z}$. If $S^{\prime}(\mathfrak{f}+a)_{b_{0}}^{M^{\prime}}$ denotes the corresponding localization of $S^{\prime}(\mathfrak{f}+\mathfrak{a})^{M^{\prime}}$ by $b_{0}=\pi(b)$, then we know that $(2.1)$ extends to an isomorphism of algebras

$$
\pi: S^{\prime}(\mathfrak{g})_{b}^{K} \rightarrow S^{\prime}(\mathfrak{f}+\mathfrak{a})_{b_{0}}^{M^{\prime}}
$$

(see Theorem 6.1, p. 147 in [3]). In particular (2.1) is injective. In this paper we describe its image when $\operatorname{dim} a=1$.

First, we recall a few basic facts about $S$-triples in $\mathrm{g}$. An $S$-triple is a set of 3 linearly independent elements $(x, e, f)$ in $g$ where $[x, e]=2 e,[x, f]=-2 f$ and $[e, f]=x$. It is called normal in case $e, f \in \mathfrak{p}$ and $x \in \mathfrak{f}$. For any $y \in \mathfrak{p}$ let $\mathfrak{p}^{y}$ denote the centralizer of $y$ in $\mathfrak{p}$. Then $y \in \mathfrak{p}$ is called regular if $\operatorname{dim} \mathfrak{p}^{y} \leqslant \operatorname{dim} \mathfrak{p}^{u}$ for any $u \in \mathfrak{p}$. Also $y \in \mathfrak{p}$ is regular if and only if $\operatorname{dim} \mathfrak{p}^{y}=\operatorname{dim} \mathfrak{a}$ (see Propositions 7 and 8, p. 770 in [2]). A normal $S$-triple $(x, e, f)$ is called principal if $e$ (and hence $f$ ) is regular. Theorem 3, p. 773 in [2] guarantee that they exist. Now fix a closed Weyl chamber $D \subset a_{\mathbf{R}}$. A normal $S$-triple $(x, e, f)$ is called standard if $e+f \in D$. Let $\Delta$ be the set of roots of $(g, a)$ and let $\Pi \subset \Delta$ be the set of simple positive roots corresponding to $D$. Also let $w$ be the unique element in $a \cap[g, g]$ such that $\langle\lambda, w\rangle=2$ for all $\lambda \in \Pi$ (obviously $w \in D$ ). From [2] we know: any normal $S$-triple is $K$-conjugate to a standard $S$-triple (Lemma 6, p. 776); a standard normal $S$-triple $(x, e, f)$ is principal if and only if $e+f=w$ (Proposition 13, p. 776); any two principal normal $S$-triples are $K_{\theta}$-conjugate (Theorem 6, p. 778).

From now on, we shall assume that $\mathfrak{a}_{\mathbf{R}} \cap\left[\mathfrak{g}_{\mathbf{R}}, \mathfrak{g}_{\mathbf{R}}\right]$ is one dimensional, that is, that $\mathfrak{g}_{\mathbf{R}}$ is of split rank one. Also $(x, e, f)$ will be a principal normal $S$-triple and $z=x / 2$.

Proposition 1. ad $z: \mathfrak{p} \rightarrow \mathfrak{p}$ is diagonalizable with eigenvalues $1,-1$ and possibly 0 .

Proof. We may assume that $(x, e, f)$ is a standard principal normal $S$-triple. One knows that $\Pi=\{\lambda\}$ and that the root space decomposition of $g$ is of the form $\mathfrak{g}=\mathfrak{g}^{0}+\mathfrak{g}^{\lambda}+\mathfrak{g}^{-\lambda}+\mathfrak{g}^{2 \lambda}+\mathfrak{g}^{-2 \lambda}$; here $\mathfrak{g}^{2 \lambda}$ and $\mathfrak{g}^{-2 \lambda}$ can be zero and $\mathfrak{g}^{0}=\mathfrak{m}+\mathfrak{a}$, where $\mathrm{m}$, as usual, is the centralizer of $\mathrm{a}$ in $\mathfrak{f}$. Therefore, the eigenvalues of $\mathrm{ad} w$, $w=e+f$, in $g$ are $2,0,-2$ and possibly 4 and -4 . Now $x$ and $w$ are $G$-conjugate, so the eigenvalues of ad $x$ in $g$ are the same as those of ad $w$. Since $\operatorname{dim}\left(p^{e} \cap[g, g]\right)=1$, $\mathfrak{p}^{e} \cap[\mathfrak{g}, \mathfrak{g}]=C e$. Therefore, up to a scalar, $e$ is the unique highest weight vector in 
$\mathfrak{p}^{e} \cap[\mathfrak{g}, \mathfrak{g}]$ of the TDS (three dimensional simple Lie algebra over $\mathbf{C}$ ) spanned by $(x, e, f)$. From the representation theory of a TDS now follows that 4 cannot be an eigenvalue of ad $x$ in $\mathfrak{p}$. For the same reason -4 cannot be a lowest weight of $x$ in $\mathfrak{p}$. Q.E.D.

Remark. The multiplicity of \pm 1 as eigenvalues of ad $z$ in $\mathfrak{p}$ is $1+\operatorname{dim} \mathfrak{g}^{2 \lambda}$. This follows immediately from the representation theory of a TDS and the fact that $[\mathfrak{f}, \mathfrak{p}] \subset \mathfrak{p}$.

Since the polynomial $b$ is $K$-invariant the closed algebraic set $V(b)=\{y \in \mathfrak{p}$ : $b(y)=0\}$ is stable under the action of $K$. Regarding its $K$-structure in the rank one case we have the following facts.

Proposition 2. The algebraic set $V(b)$ is irreducible and of codimension 1 in $\mathfrak{p}$. Moreover there are only a finite number of $K$ orbits in $V(b)$. Furthermore if $(x, e, f)$ is a principal normal $S$-triple in $\mathrm{g}$, then $K \cdot e$ and $K \cdot f$ are the unique orbits in $V(b)$ of maximal dimension so that $K \cdot e \cup K \cdot f$ is Zariski open and dense in $V(b)$.

Proof. We know that the ring $S^{\prime}(\mathfrak{p})^{K}$ of $K$-invariant polynomial functions in $\mathfrak{p}$ is a polynomial ring $C[u]$ in one homogeneous polynomial $u$ (because $\operatorname{dim} a=1$ ). Since $b$ is also homogeneous we may assume that $b=u^{r}$ for some positive integer $r$. Therefore $V(b)=u^{-1}(0)$. On the other hand we have a homomorphism of $K$ into the group of permutations of the set of irreducible factors of $u$. Since $K$ is connected each irreducible factor is $K$-invariant. This implies the irreducibility of $u$ and hence of $V(b)$. According to Theorem 9, p. 785 in [2] there are only a finite number of $K_{\theta}$-orbits, hence of $K$-orbits, in $u^{-1}(0)$, and the set of regular elements in $u^{-1}(0)$ is the unique $K_{\theta}$-orbit of maximal dimension in $u^{-1}(0)$.

Since $e$ and $f$ are nilpotent elements $b(e)=b(f)=0$ (see Corollary 5.5, p. 147 in [3]), therefore $K_{\theta} \cdot e=K_{\theta} \cdot f$ is the orbit of regular elements in $V(b)$. Without loss of generality we may assume that $(x, e, f)$ is a standard principal normal $S$-triple, so that $w=e+f$. In the rank one case under consideration, the abelian group $F$ of all elements of order 2 in $A$ is of order 2 and $F=\{1, a\}$ where $a=\operatorname{Ad}(\exp \pi i w / 2)$. If we put

$$
u=\frac{1}{2}(e-f-x) \text { and } v=\frac{1}{2}(f-e-x)
$$

then $(w, u, v)$ is a new $S$-triple. But $e=(u-v+w) / 2$, thus $a \cdot e=f$. Therefore $K_{\theta} \cdot e=K F \cdot e=K \cdot e \cup K \cdot f$. Q.E.D.

Note that all eigenvalues of $z$ in $S^{\prime}(\mathfrak{f})$ are integers and that if $S^{\prime}(\mathfrak{f})_{j}$ is the eigenspace of $S^{\prime}(\mathfrak{f})$ corresponding to the eigenvalue $j$ then

$$
S^{\prime}(\mathfrak{f})=\bigoplus_{j=-\infty}^{\infty} S^{\prime}(\mathfrak{f})_{j} .
$$

Moreover $S^{\prime}(\mathfrak{g})=S^{\prime}(\mathfrak{f}+\mathfrak{p})=S^{\prime}(\mathfrak{f}) \otimes S^{\prime}(\mathfrak{p})$, in other words an element $u \in S^{\prime}(\mathrm{g})$ can be viewed as a polynomial on $\mathfrak{p}$ with values in $S^{\prime}(\mathfrak{f})$. In particular $b$ is a polynomial on $\mathfrak{p}$ with values in $C$ and $u / b^{q}$ is a rational function on $\mathfrak{p}$ with values in $S^{\prime}(\mathfrak{f})$. 
THEOREM 3. Let $u / b^{q}\left(u \in S^{\prime}(\mathfrak{g})^{K}\right)$ be a rational function on $\mathfrak{p}$ homogeneous of degree $n \geqslant 0$. Then $u / b^{q}$ is a polynomial if and only if

$$
\frac{u}{b^{q}}(y) \in \bigoplus_{j=-n}^{n} S^{\prime}(\mathfrak{f})_{j}
$$

for all $y \in \mathfrak{p}$ such that $b(y) \neq 0$.

Proof. We can write uniquely $u=\sum u_{j}$ (finite sum) where $u_{j}$ is a polynomial function on $\mathfrak{p}$ with values in $S^{\prime}(\mathfrak{f})_{j}$. Clearly $u / b^{q}$ is a polynomial if and only if $u_{j} / b^{q}$ is a polynomial for all $j$.

Since the eigenvalues of $z$ in $\mathfrak{p}$ are $1,0,-1$ (Proposition 1) there exist

$$
\lim _{t \rightarrow+\infty} e^{-t} \operatorname{Ad}(\exp t z) \cdot y=y_{+}
$$

and

$$
\lim _{t \rightarrow-\infty} e^{t} \operatorname{Ad}(\exp t z) \cdot y=y_{-}
$$

for all $y \in \mathfrak{p}$.

If $u / b^{q}$ is a polynomial on $p$ we have

$$
\frac{u_{j}}{b^{q}}\left(y_{+}\right)=\lim _{t \rightarrow+\infty}\left(e^{-t} \operatorname{Ad}(\exp t z) \cdot y\right)=\lim _{t \rightarrow+\infty} e^{(j-n) t} \frac{u_{j}}{b^{q}}(y) .
$$

If $u_{j} \neq 0$ we can certainly choose $y \in \mathfrak{p}$ such that $u_{j}(y) \neq 0$, then (2.4) implies that $j \leqslant n$. Similarly, letting $t \rightarrow-\infty$ we obtain that $j \geqslant-n$.

Conversely if (2.3) holds and $b(y) \neq 0, y \in \mathfrak{k}$, then

$$
\lim _{t \rightarrow+\infty} \frac{u}{b^{q}}\left(e^{-t} \operatorname{Ad}(\exp t z) \cdot y\right)=\frac{u_{n}}{b^{q}}(y) .
$$

But

$$
b\left(y_{+}\right)=\lim _{t \rightarrow+\infty}\left(e^{-t} \operatorname{Ad}(\exp t z) \cdot y\right)=\lim _{t \rightarrow+\infty} b\left(e^{-t} y\right)=0,
$$

therefore $u\left(y_{+}\right)=0$ whenever $q>0$. Similarly we obtain that $u\left(y_{-}\right)=0$, for all $y \in \mathfrak{p}$ such that $b(y) \neq 0$. The element $e+f$ is $K$-conjugate to $w$, thus $e+f$ is regular and semisimple, hence $b(e+f) \neq 0$ (see $\$ 5$ in [3]). Now $(e+f)_{+}=e$ and $(e+f)_{-}=f$, thus $u(e)=u(f)=0$. Therefore $u$ vanishes on $K \cdot e \cup K \cdot f$ and hence $u$ is zero on $V(b)$ (see Proposition 2). By Hilbert's Nullstellensatz $b$ divides $u^{m}$ for some $m$. Since $b$ is a power of a prime polynomial (see the proof of Proposition 2), it follows that $u / b^{q}$ is a polynomial. Q.E.D.

If $V$ denotes a $K$-submodule of $S^{\prime}(\mathfrak{f})$ then $V_{j}$ is the eigenspace of $z$ in $V$ corresponding to the eigenvalue $j$.

LEMMA 4. Let $f \neq 0$ be a $K$-invariant rational function on $\mathfrak{p}$ with values in an irreducible $K$-submodule $V$ of $S^{\prime}(\mathfrak{f})$. Then the following conditions are equivalent:

(i) $f(y) \in \bigoplus_{j=-n}^{n} S^{\prime}(\mathfrak{f})_{j}$ for all $y \in \mathfrak{p}$ where $f$ is defined;

(ii) $V=\bigoplus_{j=-n}^{n} V_{j}$. 
Proof. That (ii) implies (i) is obvious. So assume (i) and take $v=f(y) \neq 0$, $y \in \mathfrak{p}$. Then

$$
k \cdot v=k \cdot f(y)=f(k \cdot y) \in \bigoplus_{j=-n}^{n} V_{j} \quad \text { for all } k \in K .
$$

Thus the cyclic $K$-submodule generated by $v$ is contained in $\bigoplus_{j=-n}^{n} V_{j}$. By irreducibility $V$ coincides with this cyclic module, hence $V=\bigoplus_{j=-n}^{n} V_{j}$. Q.E.D.

Now let $M$ be the connected Lie subgroup of $G$ corresponding to ad $(m)$, where $m$ denotes the centralizer of $a$ in $\mathfrak{f}$. If the rational function $f=u / b^{q}$ on $\mathfrak{p}$, $0 \neq u \in S^{\prime}(g)^{K}$, takes its values in a $K$-submodule of $S^{\prime}(\mathfrak{f})$ then the space $V^{M}$ of $M$-invariants in $V$ is different from zero. In fact, since the restriction homomorphism (2.2) is injective, there exists $y \in a$ such that $\left(u / b^{q}\right)(y)=v \neq 0$. But $k \cdot v=$ $\left(u / b^{q}\right)(k \cdot y)=\left(u / b^{q}\right)(y)=v$ for all $k \in M \subset K$.

This observation and the statements of Theorem 3 and Lemma 4 lead us to consider $K$-irreducible submodules $V$ of $S^{\prime}(\mathfrak{f})$ such that $V^{M} \neq 0$ and $V=\bigoplus_{j=-n}^{n} V_{j}$. Let $\mathrm{T}$ denote the set of all equivalence classes of irreducible holomorphic finite dimensional $K$-modules $V_{\tau}$ such that $V_{\tau}^{M} \neq 0$.

Let $H$ be the subspace of the symmetric algebra $S(\mathfrak{p})$ over $\mathfrak{p}$ spanned by all powers $e^{k}, k=0,1,2, \ldots$, of all nilpotent elements $e$ in $\mathfrak{p}$. It is clear that $H$ is a homogeneous subspace of $S(\mathfrak{p})$ and that $H$ is stable under the action of $K_{\theta}$. The elements in $H$ are called the harmonic elements in $S(\mathfrak{p})$. Now let $M_{\theta}$ be the centralizer of $a$ in $K_{\theta}$. Then $M_{\theta}=M F$ (see Lemma 20, p. 803 in [2]). Let $\Gamma$ be the set of all equivalence classes of irreducible holomorphic finite dimensional $K_{\theta}$-modules $V_{\gamma}$ such that the space $V_{\gamma}^{M_{\theta}}$ of $M_{\theta}$-invariants in $V_{\gamma}$ is different from zero. If $H_{\gamma}$ is the set of all $h \in H$ which transform under $K_{\theta}$ according to $\gamma$, then $H=\bigoplus_{\gamma \in \Gamma} H_{\gamma}$. Moreover, in the rank one case, each $H_{\gamma}$ is $K_{\theta}$-irreducible (see Theorem 2.2.9 in [1]). Since $H_{\gamma}$ is unique there exists a nonnegative integer $d(\gamma)$ such that $H_{\gamma}$ is pure homogeneous of degree $d(\gamma)$. That is, $d(\gamma)$ gives the degree in which $\gamma$ occurs harmonically in $S(\mathfrak{p})$. The remarkable fact is that $d(\gamma)$ can be obtained from the abstract $K_{\theta}$-module $V_{\gamma}$. Indeed, if $(x, e, f)$ is a principal normal $S$-triple and $z=x / 2$ then $d(\gamma)$ equals the highest eigenvalue of $z$ in $V_{\gamma}$ (Corollary 2.2.5. in [1]).

Proposition 5. Each irreducible $K$-module $V_{\tau}, \tau \in \mathrm{T}$, is isomorphic to a $\mathrm{K}$-submodule of a $K_{\theta}$-module $V_{\gamma}$ of type $\gamma \in \Gamma$.

Proof. We may assume that $K_{\theta} \neq K$; in this case the nontrivial element $a \in F$ is not in $K$. Given a $K$-module $V$ of type $\tau \in \mathrm{T}$ we define a structure of $K$-module on $V \times V$. Let $\varphi$ denote the automorphism of $K_{\theta}$ defined by conjugation by $a$. Let

$$
\gamma(k)(x, y)=(k x, \varphi(k) y) \text { and } \gamma(k a)(x, y)=(k y, \varphi(k) x)
$$

for all $(x, y) \in V \times V$ and all $k \in K$. Since $K_{\theta}=K F, \varphi(K) \subset K$ and $K \cap F=\{1\}$, $\gamma$ is well defined on $K_{\theta}$. Moreover it is easy to check that $\gamma$ is a representation of $K_{\theta}$ on $V \times V$. By hypothesis there exists $0 \neq x \in V^{M}$; then $(x, x) \in(V \times V)^{M_{\theta}}$ since $M_{\theta}=M F$ and $\varphi(M) \subset M$. Thus $(V \times V)^{M_{\theta}} \neq 0$. If $V \times V$ is $K_{\theta}$-irreducible we are done since $V$ is isomorphic to $V \times\{0\}$ as $K$-modules. If not $V \times V=W \oplus W^{\prime}$ where $W$ and $W^{\prime}$ are irreducible $K_{\theta}$-submodules ( $K_{\theta}$ is a reductive group) and we 
may assume that $W \simeq V \times\{0\}$ and $W^{\prime} \simeq\{0\} \times V$ as $K$-modules. If $W^{M_{\theta}} \neq 0, W$ is a $K_{\theta}$-module of type $\gamma \in \Gamma$ containing a $K$-submodule of type $\tau$. If not, $W^{\prime M_{\theta}} \neq 0$. Then we define $\gamma(u) w^{\prime}=\varphi(u) w^{\prime}$ for all $w^{\prime} \in W^{\prime}$ and all $u \in K_{\theta}$. The $K_{\theta}$-module $\left(W^{\prime}, \gamma\right)$ belongs to $\Gamma$ and as a $K$-module is isomorphic to $V$. Q.E.D.

The following result is of independent interest and it will be used later in this paper.

Proposition 6. When $\mathfrak{f}$ is not abelian (this means essentially $\mathfrak{g}_{\mathbf{R}} \neq \mathfrak{g} \mathfrak{l}(2, \mathbf{R})$ ) given a principal normal $S$-triple $(x, e, f)$ in $\mathrm{g}$ there exist elements $E$ and $F$ in $\mathfrak{f}$ and a real number $c$ such that $(c x, E, F)$ is an $S$-triple in $\mathrm{f}$.

Proof. Let $\mathfrak{h}_{\mathbf{R}}$ be a maximal abelian subalgebra of $\mathfrak{g}_{\mathbf{R}}$ containing $\mathfrak{a}_{\mathbf{R}}$. The complexification $\mathfrak{h}$ of $\mathfrak{h}_{\mathbf{R}}$ is a Cartan subalgebra of $\mathfrak{g}$; let $\phi$ be the set of roots of $(\mathfrak{g}, \mathfrak{h})$. Let $\sigma$ and $\tau$ denote the conjugations of $\mathfrak{g}$ with respect to $\mathfrak{g}_{\mathbf{R}}$ and $\mathfrak{u}=\mathfrak{f}_{\mathbf{R}}+i \mathfrak{p} \mathfrak{R}_{\mathbf{R}}$, respectively. If $\mathfrak{f}$ is not abelian then there exists $\alpha \in \phi$ such that $\alpha \neq \alpha^{\sigma}$ and $\left.\alpha\right|_{\mathrm{a}}=\lambda$. In fact we may assume that $\mathfrak{g}_{\mathbf{R}}$ is simple. Now if $\alpha=\alpha^{\sigma}$ for all $\alpha \in \phi$ such that $\left.\alpha\right|_{\mathfrak{a}}=\lambda$ then $\operatorname{dim} \mathfrak{g}^{\lambda}=1, \mathfrak{g}^{2 \lambda}=\{0\}$ and $\left[\mathfrak{g}^{\lambda}, \mathfrak{g}^{-\lambda}\right] \subset \mathfrak{a}$. Hence $\mathfrak{g}_{\mathbf{R}}=\mathfrak{m}_{\mathbf{R}}+\mathfrak{a}_{\mathbf{R}}+\mathfrak{g}_{\mathbf{R}}^{\lambda}$ $+\mathfrak{g}_{\mathbf{R}}^{-\lambda}$ where $\mathfrak{m}_{\mathbf{R}}=\mathfrak{m} \cap \mathfrak{g}_{\mathbf{R}}$ and $\mathfrak{g}_{\mathbf{R}}^{ \pm \lambda}=\mathfrak{g}^{ \pm \lambda} \cap \mathfrak{g}_{\mathbf{R}}$. Furthermore $\mathfrak{a}_{\mathbf{R}}+\mathfrak{g}_{\mathbf{R}}^{\lambda}+\mathfrak{g}_{\mathbf{R}}^{-\lambda}$ is an ideal in $\mathfrak{g}_{\mathbf{R}}$, thus $\mathfrak{g}_{\mathbf{R}}=\mathfrak{a}_{\mathbf{R}}+\mathfrak{g}_{\mathbf{R}}^{\lambda}+\mathfrak{g}_{\mathbf{R}}^{-\lambda}$ and $\mathfrak{f}_{\mathbf{R}}$ is one dimensional.

Take $\alpha \in \phi$ such that $\alpha^{\sigma} \neq \alpha$ and $\left.\alpha\right|_{a}=\lambda$. Then $\left(\alpha, \alpha^{\sigma}\right) \leqslant 0$ since $\alpha^{\sigma}-\alpha \notin \phi$ (Lemma 1.1.3.6, p. 25 in [4]). The quadratic form $B(X, \tau X)$ is negative definite on $\mathfrak{g}$, thus we may choose $X \in \mathrm{g}^{-\alpha}$ such that

$$
B(X, \tau X)=\frac{2}{2\left(\alpha, \alpha^{\sigma}\right)-(\alpha, \alpha)} .
$$

Let $X^{\prime}=-\tau X \in \mathrm{g}^{\alpha}$ and put $E=X+\theta X, F=X^{\prime}+\theta X^{\prime}, y=b\left(X^{\prime}+\sigma X^{\prime}\right) \in \mathfrak{g}^{\lambda}$ where $b^{2}=\left(2\left(\alpha, \alpha^{\sigma}\right)-(\alpha, \alpha)\right) /\left((\alpha, \alpha)+\left(\alpha, \alpha^{\sigma}\right)\right)<0$. Then

$$
\begin{aligned}
{[y, \theta y] } & =b^{2}\left[X^{\prime}+\sigma X^{\prime}, \theta X^{\prime}+\tau X^{\prime}\right] \\
& =b^{2}\left(\left[X^{\prime}, \tau X^{\prime}\right]+\sigma\left[X^{\prime}, \tau X^{\prime}\right]\right)
\end{aligned}
$$

since $\left[X^{\prime}, \theta X^{\prime}\right]=0$ because $\theta X^{\prime} \in \mathfrak{g}^{-\alpha^{\sigma}}$ and $\alpha-\alpha^{\sigma} \notin \phi$. But

$$
\left[X^{\prime}, \tau X^{\prime}\right]=-\left[X^{\prime}, X\right]=-B\left(X^{\prime}, X\right) H_{\alpha}=\frac{2 H_{\alpha}}{2\left(\alpha, \alpha^{\sigma}\right)-(\alpha, \alpha)},
$$

thus

$$
[y, \theta y]=\frac{2 b^{2}}{2\left(\alpha, \alpha^{\sigma}\right)-(\alpha, \alpha)}\left(H_{\alpha}+\sigma H_{\alpha}\right) \in \mathfrak{a} \cap[\mathfrak{g}, \mathfrak{g}] .
$$

Moreover

$$
\frac{2 b^{2}}{2\left(\alpha, \alpha^{\sigma}\right)-(\alpha, \alpha)} \lambda\left(H_{\alpha}+\sigma H_{\alpha}\right)=\frac{2 b^{2}\left((\alpha, \alpha)+\left(\alpha, \alpha^{\sigma}\right)\right)}{2\left(\alpha, \alpha^{\sigma}\right)-(\alpha, \alpha)}=2
$$

therefore $[y, \theta y]=w$ and $(w, y, \theta y)$ is an $S$-triple. Now if we put $x=y+\theta y$, $e=(w-y+\theta y) / 2, f=(w+y-\theta y) / 2$ we get a standard normal principal $S$ triple, since $e+f=w$. 
On the other hand if $T=[E, F]$ one can easily verify that $(T, E, F)$ is an $S$-triple. In fact,

$$
\begin{aligned}
T & =\left[X+\theta X, X^{\prime}+\theta X^{\prime}\right] \\
& =\frac{2}{2\left(\alpha, \alpha^{\sigma}\right)-(\alpha, \alpha)}\left(H_{\alpha}+\theta H_{\alpha}\right)+\left[X, \theta X^{\prime}\right]+\theta\left[X, \theta X^{\prime}\right] .
\end{aligned}
$$

Then

$$
\begin{aligned}
{[T, E]=} & \frac{2\left(-(\alpha, \alpha)+\left(\alpha, \alpha^{\sigma}\right)\right)}{2\left(\alpha, \alpha^{\sigma}\right)-(\alpha, \alpha)}(X+\theta X) \\
& +\left[\left[X, \theta X^{\prime}\right], \theta X\right]+\theta\left[\left[\mathrm{i} X, \theta X^{\prime}\right], \theta X\right]
\end{aligned}
$$

since $\left[\left[X, \theta X^{\prime}\right], X\right]=0$, because $-3 \lambda$ is not a restricted root. But

$$
\begin{aligned}
{\left[\left[X, \theta X^{\prime}\right], \theta X\right] } & =-\left[\left[\theta X^{\prime}, \theta X\right], X\right]-\left[[\theta X, X], \theta X^{\prime}\right] \\
& =\frac{2\left(\alpha, \alpha^{\sigma}\right)}{2\left(\alpha, \alpha^{\sigma}\right)-(\alpha, \alpha)} X
\end{aligned}
$$

where we used (2.5) and $[\theta X, X]=0$ since $-\alpha^{\theta}=\alpha^{\sigma}$ and $\alpha^{\sigma}-\alpha \notin \phi$. Therefore

$$
\begin{aligned}
{[T, E]=\frac{2}{2\left(\alpha, \alpha^{\sigma}\right)-(\alpha, \alpha)}\left(-(\alpha, \alpha)+\left(\alpha, \alpha^{\sigma}\right)\right)(} & X+\theta X) \\
& \left.+\left(\alpha, x^{\sigma}\right) X+\left(\alpha, \alpha^{\sigma}\right) \theta X\right)=2 E .
\end{aligned}
$$

In a similar way we obtain that $[T, F]=-2 F$.

Now

$$
x=y+\theta y=b\left(X^{\prime}+\sigma X^{\prime}+\theta X^{\prime}+\tau X^{\prime}\right)=b(F-E) .
$$

But $F-E$ is $K$-conjugate to $i[E, F]=i T$, thus $(-i / b) x$ is the semisimple element of an $S$-triple in $f$. Since any two principal normal $S$-triples are $K_{\theta}$-conjugate the proposition is proved with $c=-i / b$. Q.E.D.

THEOREM 7. Let $(x, e, f)$ be a principal normal $S$-triple in $\mathrm{g}$.

(i) If $\mathrm{g}^{2 \lambda}=0$ and $\operatorname{dim} \mathrm{g}^{\lambda}>1$ there exist $E, F \in \mathfrak{f}$ such that $(x, E, F)$ is an S-tripie in $\mathfrak{H}^{\mathrm{f}}$.

(ii) When $\mathrm{g}^{2 \lambda} \neq 0, x / 2$ is the semisimple element of an $S$-triple in $\mathrm{g}$.

Proof. By Proposition 6 we just need to compute

$$
b^{2}=\frac{2\left(\alpha, \alpha^{\sigma}\right)-(\alpha, \alpha)}{(\alpha, \alpha)+\left(\alpha, \alpha^{\sigma}\right)}=\frac{2\left(\alpha, \alpha^{\sigma}\right) /(\alpha, \alpha)-1}{1+\left(\alpha, \alpha^{\sigma}\right) /(\alpha, \alpha)} .
$$

When $\mathrm{g}^{2 \lambda}=0$ Lemma 3 in Appendix 2, p. 33 in [4] gives $\left(\alpha, \alpha^{\sigma}\right)=0$, hence $b^{2}=-1$ and we may choose $b=-i$ to get $c=1$.

If $\mathrm{g}^{2 \lambda} \neq 0$ the same lemma tells that $\left(\alpha, \alpha^{\sigma}\right)<0$, therefore $2\left(\alpha, \alpha^{\sigma}\right) /(\alpha, \alpha)=-1$, -2 or -3 . But

$$
0<\frac{4(\lambda, \lambda)}{(\alpha, \alpha)}=\frac{2 \alpha\left(H_{\alpha}+\sigma H_{\alpha}\right)}{(\alpha, \alpha)}=2+2 \frac{\left(\alpha, \alpha^{\sigma}\right)}{(\alpha, \alpha)}
$$

thus $2\left(\alpha, \alpha^{\sigma}\right) /(\alpha, \alpha)=-1$. Hence $b^{2}=-4$ and we may take $b=-2 i$ to obtain $c=1 / 2$. Q.E.D. 
Proposition 8. If $V_{\gamma}$ and $V_{\gamma^{\prime}}$ are $K_{\theta^{-}}$modules of type $\gamma, \gamma^{\prime} \in \Gamma$ both of which contain a K-submodule of the same type, then $d(\gamma)=d\left(\gamma^{\prime}\right)$.

Proof. Let $W$ be a $K$-irreducible submodule of $V_{\gamma}$. Then an $a W$ is also a $K$-submodule, hence $W \cap a W$ is equal to $W$ or to $\{0\}$ and correspondingly $V_{\gamma}=W$ or $V_{\gamma}=W \oplus a W$ since $W+a W$ is a $K_{\theta}$-submodule of $V_{\gamma}$.

Let $(x, e, f)$ be a standard principal normal $S$-triple and let $z=x / 2$. If $V_{\gamma}=W$ $\oplus a W$ then $d(\gamma)$ is the highest eigenvalue of $z$ in $W$ or in $a W$. If $z(a w)=d(\gamma) a w$ for $0 \neq w \in W$ then $(a z) w=d(\gamma) w$. But $a z=-z$ thus $z w=-d(\gamma) w$. If $\left[\mathfrak{g}_{\mathbf{R}}, \mathfrak{g}_{\mathbf{R}}\right] \neq$ $\mathfrak{S} l(2, \mathbf{R})$ then $z$ or $2 z$ is the semisimple element of an $S$-triple in $f$ and hence the eigenvalues of $z$ in a $K$-module are symmetric. Thus $d(\gamma)$ is in any case the highest eigenvalue of $z$ in $W$. When $\left[\mathfrak{g}_{\mathbf{R}}, \mathfrak{g}_{\mathbf{R}}\right]=\mathfrak{s} l(2, \mathbf{R}) \mathfrak{f}$ is abelian, $W$ is one dimensional and $d(\gamma)$ equals the absolute value of the eigenvalue of $z$ in $W$. In both cases the proposition follows. Q.E.D.

Propositions 5 and 8 enable us to define the degree of a $K$-module $V_{\tau}, \tau \in \mathrm{T}$.

Definition. The degree $d(\tau)$ of a $K$-module $V_{\tau}, \tau \in \mathrm{T}$, is the degree of any $K_{\theta}$-module $V_{\gamma}, \gamma \in \mathrm{T}$, which contains a $K$-submodule isomorphic to $V_{\tau}$.

COROLlaRy 9. Let $V_{\tau}$ be a K-module of type $\tau \in \mathrm{T}$ and let $(x, e, f)$ be a principal normal $S$-triple in $\mathfrak{q}$; put $z=x / 2$. Then $d(\tau)$ equals the highest eigenvalue of $z$ in $V_{\tau}$, when $\left[\mathfrak{g}_{\mathbf{R}}, \mathfrak{g}_{\mathbf{R}}\right] \neq \mathfrak{s} \mathfrak{l}(2, \mathbf{R})$. If $\left[\mathfrak{g}_{\mathbf{R}}, \mathfrak{g}_{\mathbf{R}}\right]=\mathfrak{s} \mathfrak{l}(2, \mathbf{R})$ then $d(\tau)$ equals the absolute value of the eigenvalue of $z$ in $V_{\tau}$.

Corollary 10 (To Proposition 5). Let $V_{\tau}$ be a $K$-module of type $\tau \in \mathrm{T}$. Then $d(\tau)$ gives the degree in which $\tau$ occurs harmonically in $S(\mathfrak{p})$.

If $\tau$ denotes an equivalence class of irreducible holomorphic finite dimensional

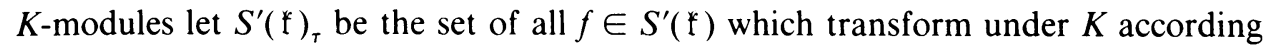
to the representation $\tau$. Since $S^{\prime}\left(\mathcal{f}^{\mathfrak{k})}\right.$ is a completely reducible $K$ module we have $S^{\prime}(\mathfrak{f})=\bigoplus_{\tau} S^{\prime}(\mathfrak{f})_{\tau}$.

THEOREM 11. Let $u / b^{q}\left(u \in S^{\prime}(a)^{\kappa}\right)$ be a rational function on $p$ homogeneous of degree $n \geqslant 0$. Then $u / b^{4}$ is a polynomial if and only if

$$
\frac{u}{b^{q}}(y) \in \bigoplus_{\substack{\tau \in \mathrm{T} \\ d(\tau) \leqslant n}} S^{\prime}\left({ }^{*}\right)_{\tau}
$$

for all $y \in \mathfrak{p}$ where $b(y) \neq 0$.

Proof. According to Theorem 3 we have to prove that (2.3) is satisfied if and only if (2.6) is true. Assume (2.3). Right after Lemma 4 we observed that

$$
\frac{u}{b^{q}}(y) \in \bigoplus_{\tau \in \mathrm{T}} S^{\prime}(\mathfrak{f})_{\tau} .
$$

Now Lemma 4 and Corollary 9 imply that

$$
\frac{u}{b^{q}}(y) \in \bigoplus_{\substack{\tau \in \mathrm{T} \\ d(\tau) \leqslant n}} S^{\prime}(\mathfrak{f})_{\tau} .
$$

Conversely if (2.6) is verified then (2.3) follows immediately. Q.E.D. 
The group $M^{\prime}$ leaves $\mathfrak{f}+$ a invariant, thus the Weyl group $W=M^{\prime} / M$ operates canonically on the ring $S^{\prime}(\mathfrak{f}+\mathfrak{a})^{M}=S^{\prime}(\mathfrak{f})^{M} \otimes S^{\prime}(a)$ of $M$ invariants in $S^{\prime}(\mathfrak{f}+\mathfrak{a})$. Let $\left(S^{\prime}(\mathfrak{f})^{M} \otimes S^{\prime}(\mathfrak{a})\right)^{W}$ denote the ring of Weyl group invariant elements in $S^{\prime}(\mathfrak{f})^{M}$ $\otimes S^{\prime}(a)$. We are ready to state and prove our main theorem.

THEOREM 12. The operation of restriction from $g$ to $\mathfrak{f}+$ a induces an isomorphism of $S^{\prime}(\mathrm{g})^{K}$ onto

$$
\bigoplus_{n \geqslant 0} \bigoplus_{\substack{\tau \in \mathrm{T} \\ d(\tau) \leqslant n}}\left(S^{\prime}(\mathfrak{f})_{\tau}^{M} \otimes S_{n}^{\prime}(\mathfrak{a})\right)^{W} .
$$

Proof. We already know that the restriction homomorphism (2.1) is injective and that its image is contained in $S^{\prime}(\mathfrak{f}+\mathfrak{a})^{M^{\prime}}=\left(S^{\prime}(\mathfrak{f})^{M} \otimes S^{\prime}(a)\right)^{W}$. Theorem 11 now shows, more precisely, that the image is contained in (2.7). Let $v \in$ $\bigoplus_{\tau \in \mathrm{T}, d(\tau) \leqslant n}\left(S^{\prime}(\mathfrak{f})_{\tau}^{M} \otimes S_{n}^{\prime}(a)\right)^{W}$. Since (2.2) is an isomorphism of algebras there exist $u \in S^{\prime}(g)^{K}$ and $q \geqslant 0$ such that $\pi\left(u / b^{q}\right)=v$. By the $K$ invariance of $u / b^{q}$ we have that

$$
\frac{u}{b^{q}}(y) \in \bigoplus_{\substack{\tau \in \mathrm{T} \\ d(\tau) \leqslant n}} S^{\prime}(\mathfrak{f})_{\tau}
$$

for all $y \in K \cdot \mathfrak{a}(K \cdot \mathfrak{a}$ contains the set of all $y \in \mathfrak{p}$ where $b(y) \neq 0)$. On the other hand since $K \cdot \mathfrak{a}$ is dense in $\mathfrak{p}, u / b^{q}$ is homogeneous of degree $n$ on $\mathfrak{p}$. Then Theorem 11 tells us that $u / b^{q} \in S^{\prime}(g)^{K}$. This completes the proof of the theorem. Q.E.D.

\section{REFERENCES}

1. B. Kostant, On the existence and irreducibility of certain series of representations, Lie Groups and their Representations, 1971 Summer School in Math. (I. M. Gelfand, editor), Wiley, New York, 1975, pp. 231-330.

2. B. Kostant and S. Rallis, Orbits and representations associated with symmetric spaces, Amer. J. Math. 93 (1971), 753-809.

3. B. Kostant and J. Tirao, On the structure of certain subalgebras of a universal enveloping algebra, Trans. Amer. Math. Soc. 218 (1976) 133-154.

4. G. Warner, Harmonic analysis on semi-simple Lie groups. I, Springer-Verlag, New York, 1972.

5. J. Lepowsky, Algebraic results on representations of semisimple Lie groups, Trans. Amer. Math. Soc. 176 (1973), 1-44.

6. J. Dixmier, Algèbres enveloppantes, Gauthier-Villars, Paris, 1974.

I.M.A.F., Universidad Nacional de Córdoba, 5000 Córdoba, Argentina 